\title{
STEM EBIC to Study 2D Materials
}

E. R. White ${ }^{1}$, Alex Kerelsky ${ }^{1}$, Grant Jasmin ${ }^{1}$, William A. Hubbard ${ }^{1}$, Matthew Mecklenburg ${ }^{2}$, and B. C. Regan $^{1}$

1. Department of Physics and Astronomy and California NanoSystems Institute, University of California, Los Angeles, California 90095 USA

2. Center for Electron Microscopy and Microanalysis, University of Southern California, Los Angeles, California 90089 USA

Since its discovery in 2004 graphene has become a very widely studied material. The electronic excitations in single layer graphene can be described as two-dimensional massless Dirac particles, resulting in desirable electronic properties for nanoelectronic devices. Adding layers allows the electronic properties to be tuned, thus multilayer graphene is better suited for some device applications. Characterizing defects in this material is critical for understanding device performance. Recently Butz et al. have used dark field TEM to image dislocations in bilayer graphene, furthering our understanding of its electronic properties and the key importance of defects [1]. Here, we report using electron beam induced current (EBIC) measurements in a scanning transmission electron microscope (STEM) to image local changes in the conductivity of multilayer graphene.

EBIC is a well-established electron microscopy imaging technique that is typically used to characterize semiconductor devices $[2,3]$. As the electron beam rasters across the sample the current induced by the beam at each pixel is measured. This EBIC signal is digitized in synchronicity with the signal from the HAADF detector, producing a spatial map of the beam induced current and the usual STEM image simultaneously. When the incident electron beam impacts a semiconducting material it creates electron-hole pairs by inelastically scattering valence electrons into the conduction band. These charge carriers are collected by an electric field, creating the current that is the EBIC signal. Each incident electron produces many electron-hole pairs, resulting in typical EBIC signals on the order of nanoamperes. Another, much weaker way to induce current in the sample is by secondary electron emission. Occasionally a high-energy incident electron will collide with an electron in the sample, ejecting it into the vacuum. The resulting net positive charge on the sample escapes to ground via the current amplifier, creating the EBIC signal. In this case no gain results and the currents are smaller than the incident beam current, typically picoamperes.

Figure 2 shows a single, multilayer graphene sheet and the corresponding ADF and EBIC images. The contrast in the EBIC image is not produced by electron-hole separation. Electron-hole pairs generated in the graphene must recombine since there is no second electrode to collect the opposite charge carrier. Thus, we attribute the EBIC signal to secondary electron emission. The incident electron beam current is $200 \mathrm{pA}$ and the EBIC signal generated in the graphene is about $10 \mathrm{pA}$, which is consistent with expectations for a secondary electron EBIC signal. A comparison of the ADF and EBIC images in Figure 2 reveals the advantages of EBIC imaging. Regions of low conductivity in the graphene sheet likely caused by defects give a smaller EBIC signal. Contrast from these defects is completely absent from the ADF image. The ADF signal is dominated by residue left over from the graphene transfer process. EBIC is sensitive to the electrical properties of the sample, and thus reveals the important electrical information desired when characterizing devices for nanoelectronic applications. 


\section{References:}

[1] B Butz et al, Nature 505 (2014) p. 533. [2] H

Leamy, J. Appl. Phys. 53 (1982) p. R51

[3] K Bunker et al, Mat. Res. Soc. Symp. Proc. 743 (2003) p L10.10.1

[4] This work is supported by STARnet, a Semiconductor Research Corporation program sponsored by MARCO and DARPA, by NSF DMR award 1206849, and the Electron Imaging Center for

NanoMachines at UCLA.

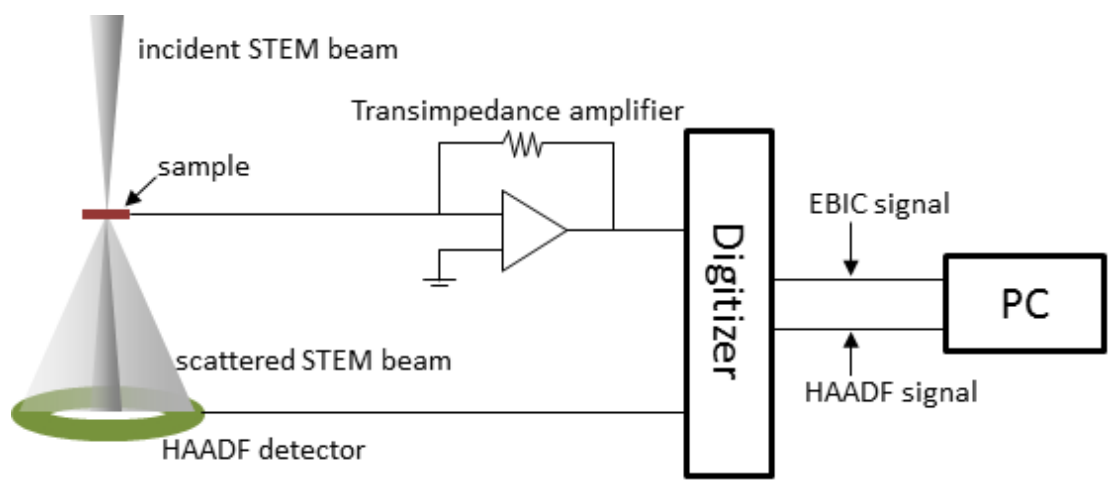

Figure 1. Schematic of simultaneous EBIC and HAADF data acquisition. Current generated in the sample is converted to a voltage appropriate for the microscope digitizing electronics by the transimpedance amplifier. The HAADF signal is digitized in parallel with the EBIC signal, thus the two images are generated simultaneously.
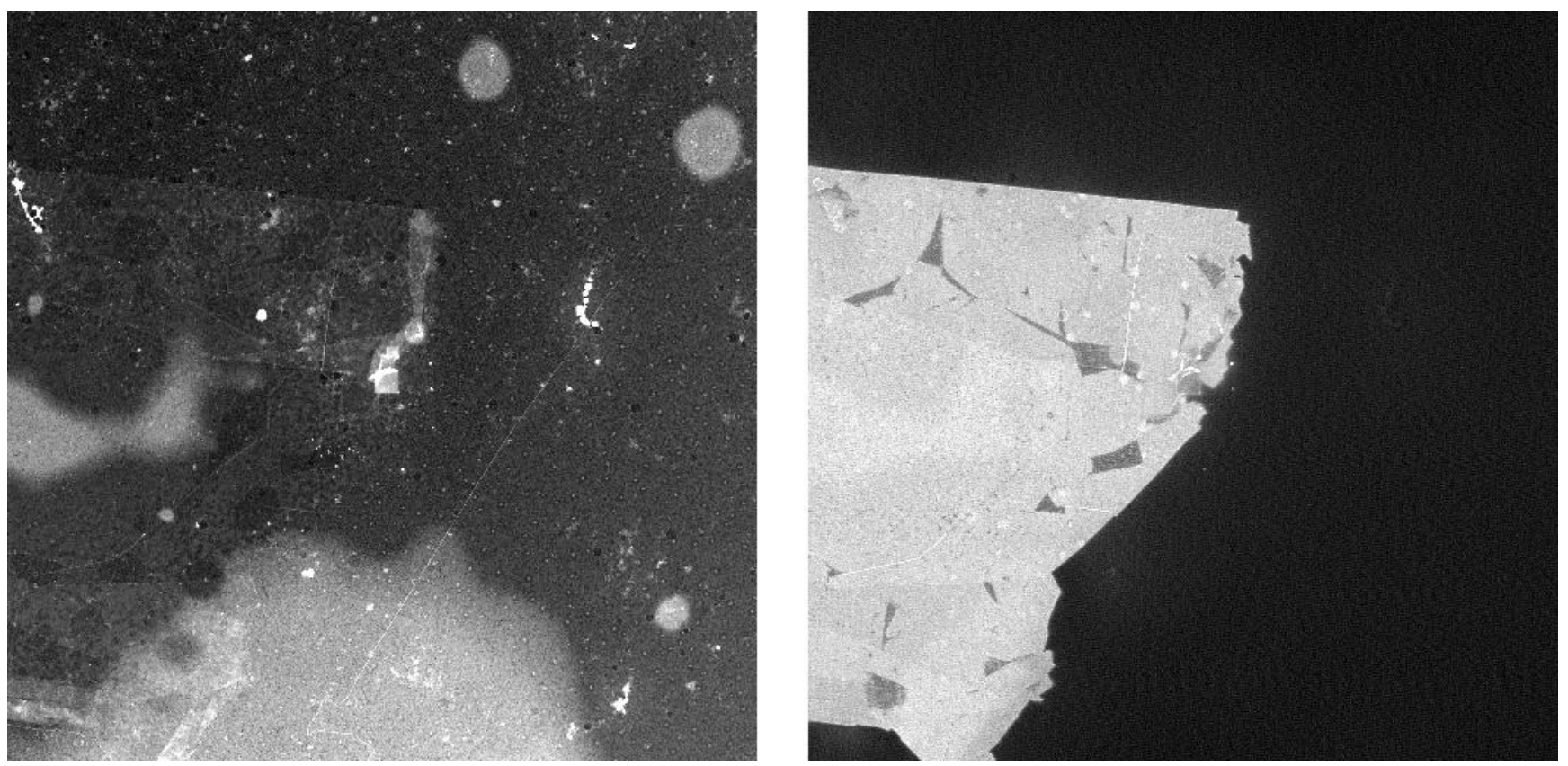

Figure 2. ADF (left) and EBIC (right) images of a multilayer graphene sheet. In the ADF image residue left from the graphene transfer process produces most of the contrast. EBIC signal is only generated by electrically connected regions, hence only the graphene generates contrast. Areas with reduced EBIC signal in the graphene sheet correspond to regions of reduced conductivity. The images are 7 um on a side. 